\begin{tabular}{c} 
International Journal of Engineering \& Technology, 7 (3.17) (2018) 76-84 \\
International Journal of Engineering \& Technology \\
SPC \\
Website: www.sciencepubco.com/index.php/IJET \\
Research paper \\
\hline
\end{tabular}

\title{
Applicability of A Rotary Eddy Current Damper in Passenger Vehicle Suspension with Parallel Inerter
}

\author{
Ming Foong Soong ${ }^{1,2}$, Rahizar Ramli ${ }^{1 *}$, Ahmad Abdullah Saifizul ${ }^{1}$ and Mahdieh Zamzamzadeh $^{1}$ \\ ${ }^{1}$ Advanced Computational and Applied Mechanics (ACAM) Research Group \\ Department of Mechanical Engineering, Faculty of Engineering \\ ${ }^{2}$ Centre for the Initiation of Talent and Industrial Training (CITra) \\ University of Malaya, 50603 Kuala Lumpur, Malaysia \\ *Corresponding author Email: rahizar@um.edu.my
}

\begin{abstract}
Numerous studies have proven that the performance of vehicle suspension can be benefited by an inerter in parallel to conventional spring-damper setup, yet its usability in passenger vehicle suspension is still limited by practical consideration in physical implementation. One way of achieving better physical implementation of the parallel inerter suspension layout is to exploit the inerter's flywheel as a metallic conductor to integrate passive damping in the form of a rotary eddy current damper. However, the feasibility of eddy current damping in this specific application remains unknown. This study investigates the applicability of eddy current damping incorporated in an inerter in terms of the achievable damping rates as required in typical passenger vehicle suspensions. In the study, passive eddy current damping due to constant magnetic field around the flywheel of a mathematically designed inerter was computed through simulation, and the range of achievable damping rates due to parametric variations, for instance air gap and magnetic coverage, was evaluated. Results of the parametric analysis showed that the induced eddy current damping from a rack-and-pinion inerter's flywheel, considering the designed inertance as prerequisite, was at least capable of achieving $1500 \mathrm{Nsm}^{-1}$. As the achievable damping was within the range of suitable damping rates for typical passenger vehicles, rotary eddy current damper was deemed applicable in passenger vehicle suspension employing parallel inerter.
\end{abstract}

Keywords: Eddy current damper, Electromagnetic damping, Vehicle suspension, Inerter, Parametric analysis.

\section{Introduction}

In essence, a typical suspension system consists of both a spring element and a damping element to provide vibration isolation to a sprung mass. The spring supports the static load of the sprung mass and stores the system energy due to vibrations, while the damper dissipates the energy to dampen the sprung mass response. For a vehicle suspension, the working of spring and damper serves the important purposes of isolating the vehicle body from ground vibrations for good ride performance, as well as maintaining consistent tire-road contact for good road holding performance [1]. In the recent decade, numerous studies have shown that the ride performance of a vehicle suspension can be enhanced by an additional suspension element called the inerter [2-7]. The inerter is a two-terminal suspension element like spring and damper, but with the property that the force applied at its two terminals is directly proportional to the relative acceleration between the terminals [8], such that:

$F=b\left(a_{2}-a_{1}\right)$

in which $F$ is the inerter force, $a_{1}$ and $a_{2}$ are the accelerations of the terminals, and $b$ is a proportionality constant known as the inertance which is again analogous to other suspension properties like spring stiffness and damping rate. The inerter is essentially a two-terminal mass that is achieved through the use of a rotating flywheel and some mechanisms, such as ball-screw and rack-and- pinion, to achieve the conversion between rotational motion of the flywheel and the translational suspension deflection.

Although the ride performance benefit brought by an inerter in vehicle suspension has been well-proven in the above mentioned studies, its usability in passenger vehicle suspension is currently limited due to practical consideration in its physical implementation. While a physical inerter device is definitely possible as demonstrated experimentally [9], its actual addition on passenger vehicle suspension is less practical considering the space constraint around the vehicle suspension system. To elaborate, with the parallel inerter layout, it is not realistic to directly add an inerter in parallel to existing spring and damper due to physical space limitation. One way of solving this issue of parallel inerter is to integrate into it the required passive damping in the form of a rotary eddy current damper. Eddy current damping is a specific form of electromagnetic damping in which the energy from vibration is dissipated due to the flow of eddy current in a metallic conductor under electromagnetic induction. This form of passive damping has been proven to be applicable in passenger vehicle suspension [10]; however, the applicability is only valid for linear eddy current damper. Meanwhile, rotary eddy current damper in passenger vehicle suspension has unfortunately never been studied in depth, especially in the context of this specific incorporation in a parallel inerter.

Following this, it appears important that this study investigates the applicability of rotary eddy current damper in passenger vehicle suspension considering parallel inerter, in terms of the achievable damping rates. In the study, rotary eddy current damping was 
incorporated in an inerter by introducing constant magnetic field to the rotating flywheel which served as a metallic conductor. Through a comprehensive parametric analysis involving variations of several electromagnetic-related parameters, it was found that the integrated eddy current damping was capable of achieving suitable damping rates for typical passenger vehicles, and thus its applicability in passenger vehicle suspension was confirmed.

\section{An Overview of Eddy Current Damping}

From the energy point of view, the damper in a suspension system functions as an energy dissipater as it dissipates the unwanted vibrational kinetic energy of the system to the surrounding. In a passive vehicle suspension, conventionally a fluid damper is used, and damping is achieved through the viscosity of the fluid within the damper. For fluid or viscous damper, the kinetic energy is converted to thermal energy, and later dissipated as heat, due to the resistance to motion as a result of the fluid viscosity. Besides viscous damping, another way of achieving damping effect is to utilize electromagnetic induction to convert the unwanted kinetic energy from the system's vibration or movement to electrical energy before extracting the electrical energy out from the system. This type of damping is generally known as electromagnetic damping [11]. Eddy current damping is basically a specific form of electromagnetic damping in which the converted electrical energy is simply dissipated due to eddy current. For an eddy current damper, the induced electrical current, in this case the eddy current, simply flows in an electrical conductor [12], causing the converted electrical energy to be dissipated through resistive heating.

In the simplest description, eddy current is the current induced in a conductive material in the presence of a magnetic field. Due to the electrical resistance, the induced current will be dissipated into heat at the rate of $I^{2} R$ [13], in which $I$ is the current and $R$ is the resistance of the conducting material. Dynamically, the interaction of eddy current with the magnetic field will result in a reaction force [14]:

$$
F=J \times B
$$

in which $\boldsymbol{F}$ is the Lorentz force, $\boldsymbol{J}$ is the current density which is representative of the induced eddy current, and $\boldsymbol{B}$ is the magnetic flux density of the externally applied magnetic field. When acting in a suitable direction, this serves as the damping force to the system as it opposes the system's motion.

The potential of eddy current damping to be used as a source of passive damping in vibration systems can be shown by some fundamental studies. For instance, the damping contributed by eddy current in the well-known vibrating cantilever beam problem has been investigated in the past $[13,15]$. These studies tested single and multiple permanent magnets that were mounted near a conducting sheet attached to a cantilever beam to evaluate the beam's response. It was determined that the presence of eddy current damping increased the damping ratio significantly compared to the structure's initial damping and thus provided sufficient damping force to quickly suppress the beam's vibration. For the above studies, the resulted eddy current damping effect acted as passive damping due to the constant magnetic field provided by permanent magnets. However, extending from the above, variable damping for an eddy current damper has also been shown possible when the magnetic flux density is varied, either through controlled positioning of magnetic source [16] or by directly variable magnetic source [17].

In the scope of passenger vehicle suspension, the applicability of eddy current damping has only been recently studied [10]. In this relevant study, a linear eddy current damper, consisting of a magnet-slotted mover and a hollow cylindrical aluminum conductor, was compared to existing vehicle suspension dampers to analyze its achievable damping. It was shown that a full-scale eddy current damper attained comparable damping force in compression, but less in extension, relative to fluid dampers. In a later study [18], the linear eddy current damper was applied as a source of passive damping, auxiliary to an active electromagnetic damper, to form a hybrid electromagnetic damper, with the intention that the passive eddy current damping provided reliability to the active electromagnetic damper in the case of power breakdown. Similarly, the study stated that by introducing eddy current damping to the hybrid electromagnetic damper, a passive damping typical of passenger vehicle application, approximately $1570 \mathrm{Nsm}^{-1}$, can be achieved to act as fail-safe guarantee to the active part.

\section{Feasibility of Eddy Current Damping in Vehicle Suspension}

It can be seen that the capability of eddy current to provide damping effect has been proven for fundamental vibration problem as well as specifically to passenger vehicle suspension application. However, it must be noted that the proven feasibility strictly only points to linear eddy current damper. Meanwhile, rotary eddy current damper has, at best, only been explored remotely as electromagnetic brake [14] which obviously has vastly different requirements. The use of rotary eddy current damping as suspension damper has never been evaluated before, never mind the consideration of its use along with a parallel inerter.

In this study, the applicability of rotary eddy current damper in the form of achievable damping rates was investigated, with the consideration of an interesting concept that the damping was incorporated in an inerter for parallel suspension layout. Conceptually, rotary eddy current damping can be conveniently achieved in a typical flywheel-based inerter (Figure 1(a)) by exploiting the rotating flywheel, which is usually metallic, as the electrical conductor for eddy current induction in the presence of magnetic source such as permanent magnets (Figure 1(b)). While this concept provides the fundamental feasibility, its suitability to be used in passenger vehicle suspension, or the applicability, is worth analyzed as described subsequently.

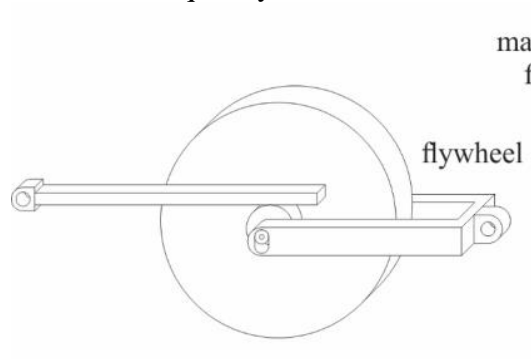

(a)

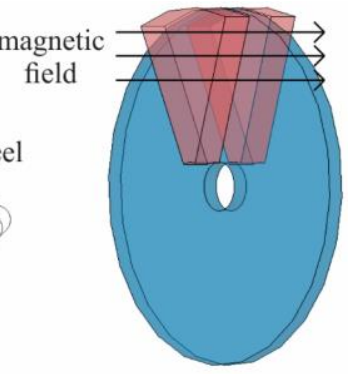

(b)
Fig. 1: (a) The schematic diagram of a typical flywheel-based inerter, and (b) the incorporation of rotary eddy current damping through the addition of permanent magnets

\subsection{Mathematical Design of Inerter}

A particularly crucial prerequisite for this applicability study on rotary eddy current damper is the predetermination of the flywheel's dimensional parameter values based on the required inertance of the inerter. The determination of required inertance for optimum vehicle ride performance can be carried out using the two-degree-of-freedom quarter vehicle model as represented schematically in Figure 2 and mathematically by (3) and (4):

$$
\begin{aligned}
& m_{s} \ddot{z}_{s}=k\left(z_{u}-z_{s}\right)+c\left(\dot{z}_{u}-\dot{z}_{s}\right)+b\left(\ddot{z}_{u}-\ddot{z}_{s}\right) \\
& m_{u} \ddot{z}_{u}=k_{t}\left(z_{g}-z_{u}\right)-k\left(z_{u}-z_{s}\right)-c\left(\dot{z}_{u}-\dot{z}_{s}\right)-b\left(\ddot{z}_{u}-\ddot{z}_{s}\right)
\end{aligned}
$$




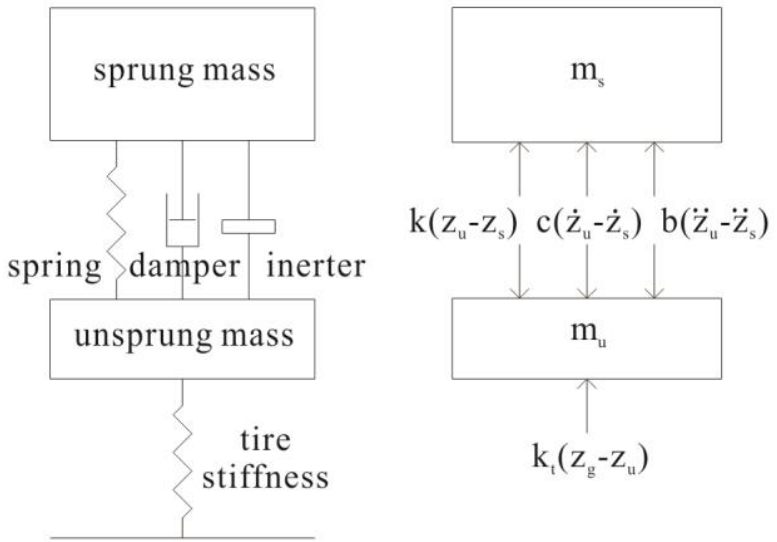

Fig. 2: The schematic representation of a quarter vehicle model

in which $m_{s}, m_{u}$ are the sprung and unsprung mass values, $z_{s}, z_{u}$ are the vertical sprung and unsprung mass displacements, $z_{g}$ is the ground displacement due to road input, $k_{t}$ is the tire's vertical stiffness, while $k, c, b$ are the suspension's spring stiffness, damping rate and inertance. In the study, a quarter vehicle model with the following passenger vehicle parameters was considered: $m_{s}=$ $317.5 \mathrm{~kg}, m_{u}=45.4 \mathrm{~kg}, k=22000 \mathrm{Nm}^{-1}, c=1500 \mathrm{Nsm}^{-1}$, and $k_{t}=$ $192000 \mathrm{Nm}^{-1}$. Meanwhile, for inertance, a wide range from $b=0$ $\mathrm{kg}$ to $b=320 \mathrm{~kg}$ was tested, similar to a relevant past study on vehicle suspension with parallel inerter [5]. By analyzing the vehicle responses due to a $0.1 \mathrm{~m}$ step road input and a random smooth road input with the stated range, the required inertance was determined to be $b=6 \mathrm{~kg}$, based on optimum ride performance which translated into minimum root-mean-squared sprung mass acceleration. This inertance value became the basis for the subsequent determination of the flywheel's dimensional parameter values. In selecting appropriate dimensional parameter values of the flywheel of inerter, two most commonly used rotational-totranslational conversion mechanisms in an inerter were considered, namely the ball-screw mechanism and the rack-and-pinion mechanism. Both resulted inerter types are still flywheel-based, and the core element (flywheel) which gives rise to inertance remains the same. Assuming the typical cylindrical flywheel structure, the equivalent inertances due to design parameters of a ball-screw inerter and a basic rack-and-pinion inerter with only a single pinion were respectively derived to be:

$b=\pi^{3} \rho t\left(d_{o}{ }^{4}-d_{i}{ }^{4}\right) / 8 p$

$b=\pi \rho t\left(d_{o}{ }^{4}-d_{i}{ }^{4}\right) / 8 d_{p}{ }^{2}$

in which $d_{o}$ is the flywheel's outer diameter, $d_{i}$ is the inner diameter (for a hollow cylindrical flywheel structure), $t$ is the thickness of the flywheel, $\rho$ is the flywheel's material density, $p$ is the pitch of the ball-screw for this mechanism, and $d_{p}$ is the pinion diameter of the basic rack-and-pinion mechanism. It can be seen that most design parameters are related to the dimensions of the flywheel. For these design parameters, suitable ranges of values were assigned as in Table 1 .

Table 1: Ranges for the Design Parameters

\begin{tabular}{|c|c|}
\hline Parameter & Range of Values \\
\hline Outer diameter, $d_{o}(\mathrm{~m})$ & {$[0.100-0.150]$} \\
\hline Inner diameter, $d_{i}(\mathrm{~m})$ & {$[0.010-0.020]$} \\
\hline Thickness, $t(\mathrm{~m})$ & {$[0.005-0.020]$} \\
\hline Density, $\rho\left(\mathrm{kgm}^{-3}\right)$ & {$[2700,8900,7800]$} \\
\hline $\begin{array}{c}\text { Pitch, } p(\mathrm{~m}) \\
\text { (ball-screw only) }\end{array}$ & {$[0.002-0.010]$} \\
\hline $\begin{array}{c}\text { Pinion diameter, } d_{p}(\mathrm{~m}) \\
\text { (rack-and-pinion only) }\end{array}$ & {$[0.020-0.040]$} \\
\hline
\end{tabular}

These ranges were determined based on reasonable implementation in passenger vehicle suspension. For instance, the range of outer diameters was selected by considering typical diameters of a suspension strut as well as the need of including a cylindrical fly- wheel in the setup. Meanwhile, the millimeter range and centimeter range of the ball-screw pitch and pinion diameter were meant to emulate commonly available ball-screws and pinions for the respective mechanisms. Lastly, the discrete density values of the flywheel were based on three common metallic materials, namely aluminum, copper and steel. With the exception of steel that was considered due to it being a widely available conducting material, the other metallic materials were considered as they were specifically employed in eddy current induction due to their very high electrical conductivities.

Using these ranges, a comprehensive exploration of the design space was carried out, obviously with the goal of finding the suitable set of $\left[d_{o}, d_{i}, t, \rho, p\right]$ and $\left[d_{o}, d_{i}, t, \rho, d_{p}\right]$ such that the required $b=6 \mathrm{~kg}$ was obtained. Secondarily, a small flywheel mass should also be fulfilled, since an ideal inerter should be massless, similar to other suspension elements like spring and damper. Assuming cylindrical flywheel, the mass $m$ was easily derived as:

$m=\pi \rho t\left(d_{o}{ }^{2}-d_{i}^{2}\right) / 4$

Since the inertance and mass definitions were directly computable, it was possible to determine every combination of parameter values within the ranges for a sufficiently fine resolution. Of the many combinations of parameter values (design points), a design point was selected each for ball-screw type of inerter and rackand-pinion type of inerter. For the former, the selected point was: $d_{o}=0.123 \mathrm{~m}, d_{i}=0.017 \mathrm{~m}, t=0.005 \mathrm{~m}, \rho=2700 \mathrm{kgm}^{-3}$ (aluminum), and $p=0.002 \mathrm{~m}$ which resulted in $b=5.9858 \mathrm{~kg}$ and $m=$ $0.1573 \mathrm{~kg}$. Meanwhile, for the latter, the desirable point was: $d_{o}=$ $0.146 \mathrm{~m}, d_{i}=0.020 \mathrm{~m}, t=0.005 \mathrm{~m}, \rho=2700 \mathrm{kgm}^{-3}$ (aluminum), and $d_{p}=0.020 \mathrm{~m}$ which resulted in $b=6.0199 \mathrm{~kg}$ and $m=0.2218$ $\mathrm{kg}$. With the required inertance fulfilled, these two sets of design parameter values, especially the dimensional values, would then be adopted in rotary eddy current damper analysis.

\subsection{Electromagnetic-Related Parametric Analysis}

In the applicability analysis of rotary eddy current damper in passenger vehicle suspension, the damping characteristics and, most critically, the achievable rates of linear passive damping from the incorporated rotary eddy current damping were evaluated. Describing in general, this was done by solving computationally the situation of rotary eddy current damping due to the presence of constant magnetic field to obtain the rotational damping characteristics for some design configurations (parametric variations) before deriving the linear damping rates.

The parametric analysis considered a fundamental rotary eddy current damper model, as shown in Figure 3, to represent the incorporation of eddy current damping using the cylindrical flywheel of an inerter. The model was made up of the cylindrical aluminum flywheel as the electrical conductor, based on the material density and dimensional parameter values determined previously for the fulfillment of inertance, and a pair of high-magneticstrength permanent magnets as the constant magnetic source. Although the modeling was clearly a basic one and by no means served as a representation of the detailed physical construction, it was deemed adequate for applicability study as the model still captured the fundamental working principle of the incorporated rotary eddy current damper. 

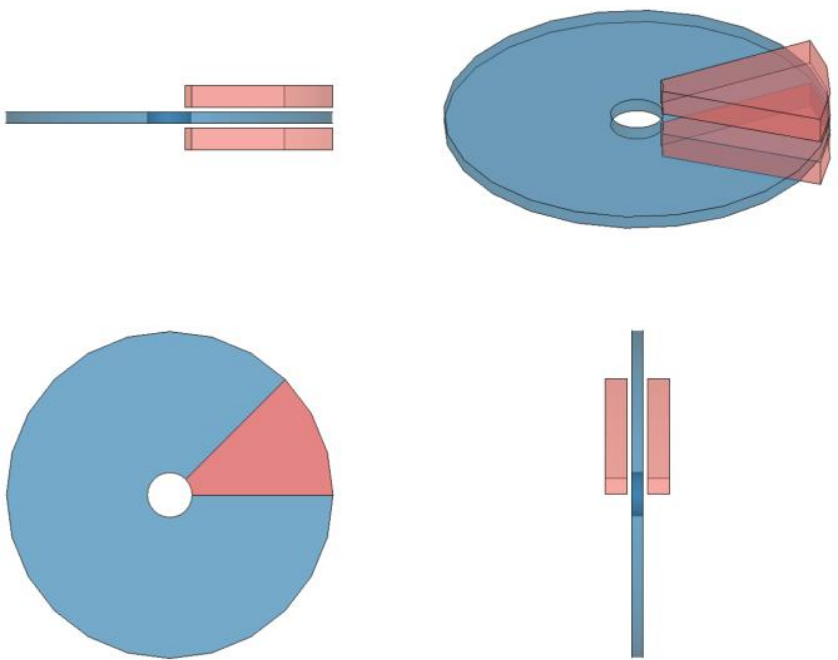

Fig. 3: Illustration of the rotary eddy current damper model
For this parametric analysis, a total of seven electromagneticrelated eddy current damper design parameters were considered, namely (i) air gap between magnet and conductor, (ii) magnet configuration (poles' arrangement), (iii) type of coverage of the magnetic source, (iv) magnet material, (v) height of magnet, (vi) number of magnet segments, and (vii) physically allowable shape of magnet for this model. These electromagnetic-related design parameters were expected to have effects on the intensity of eddy current induction and consequently on the achievable eddy current damping. Concerning these seven parameters, comprehensive ranges of values or selections were taken into consideration in order to have comprehensive parametric variations. These are stated in Table 2.

Table 2: Variations of Electromagnetic-related Parameters

\begin{tabular}{|c|c|c|c|c|c|c|}
\hline \multicolumn{7}{|c|}{ Parameter } \\
\hline (i) & (ii) & (iii) & (iv) & (v) & (vi) & (vii) \\
\hline $2 \mathrm{~mm}$ & Opposite poles & Partial & Neodymium-iron-boron (NdFeB) & $5 \mathrm{~mm}$ & 1 segment & Arc \\
\hline $6 \mathrm{~mm}$ & Single pole & & & $15 \mathrm{~mm}$ & 4 segments & \\
\hline $8 \mathrm{~mm}$ & & & & $20 \mathrm{~mm}$ & & \\
\hline $10 \mathrm{~mm}$ & & & & & & \\
\hline
\end{tabular}

Of the many possible combinations of parameter values or selections, a reasonable one was taken as the reference design or base design. This involved the following: (i) $2 \mathrm{~mm}$ of air gap, (ii) magnet pair with opposite poles facing each other (for mostly axiallyoriented magnetic field), (iii) partial magnetic field coverage, (iv) neodymium-iron-boron $(\mathrm{NdFeB})$ as magnet material (strong magnetic field strength), (v) $10 \mathrm{~mm}$ magnet height, (vi) single magnet segment, and finally (vii) the original arc magnet shape as in Figure 3. In addition to this electromagnetic-related base design, both ball-screw and rack-and-pinion rotational-to-translational conversion ratios, as determined from the selected pitch and pinion diameter previously, were also considered. Starting with the base design, the model was repetitively solved using ANSYS $^{\circledR}$ Maxwell to compute the opposing or damping torques acting on the moving flywheel for a wide range of constant rotational speeds. This allowed the determination of the complete torque-speed characteristic, which was effectively the rotational damping characteristic for the base design. The same approach was then executed for each parametric variation, in which each concerned parameter in Table 2 was varied while retaining others at the base values or selections. Finally, through the respective rotational-totranslational conversion ratios as mentioned above, the achievable linear damping due to rotary eddy current damper model was determined as achievable damping rates.

\subsection{Achievable Damping Characteristics and Damping Rates}

With the computational work outputting the necessary numerical results, the analysis on the applicability of rotary eddy current damping in passenger vehicle suspension could be carried out. Firstly, the torque-speed characteristics due to rotary eddy current damping, considering ball-screw and rack-and-pinion conversion mechanisms, were obtained as shown in Figure 4(a) and Figure 4(b) respectively. 
Torque-speed Characteristic for Eddy Current Dampe with Ball-screw Mechanism

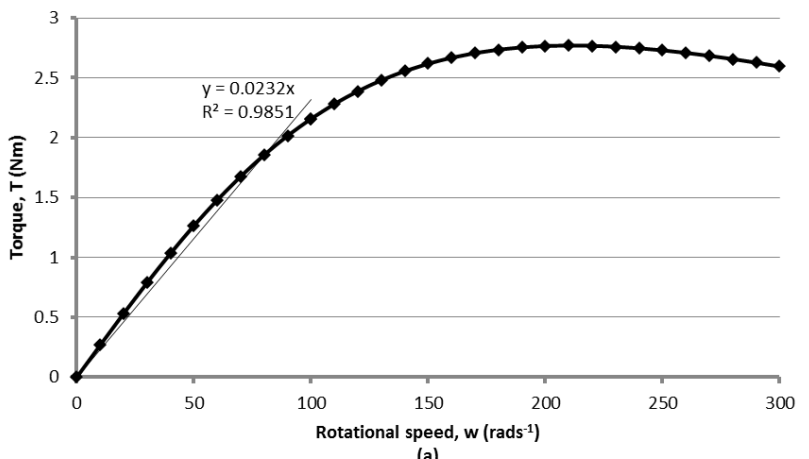

Torque-speed Characteristic for Eddy Current Damper with Rack-and-pinion Mechanism

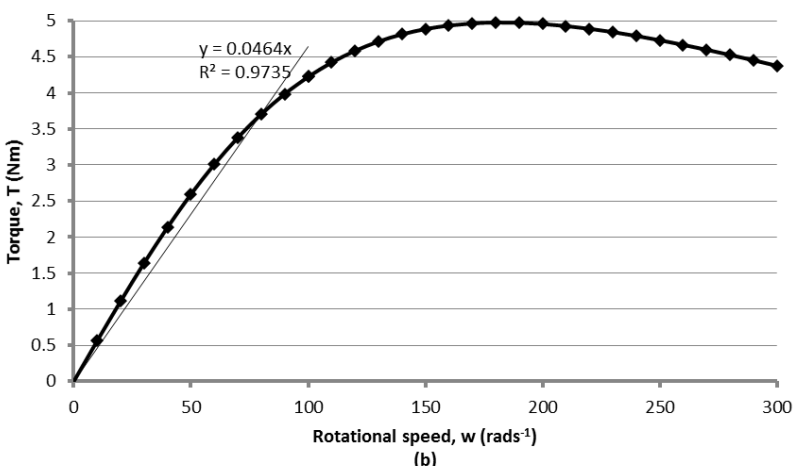

(b)

Fig. 4: The torque-speed characteristic due to eddy current damping considering (a) ball-screw mechanism and (b) rack-and-pinion mechanism of the inerter

Because the torque-speed characteristic is effectively the rotational damping characteristic of a rotary eddy current damper, Figure 4 basically describes the damping curve unique to eddy current damping. Generally, the complete eddy current damping curve is non-linear and consists of two regions separated by a maximum point. In the beginning region, the damping effect is mostly linear, because the induced eddy current in the electrical conductor under the presence of a constant external magnetic field varies only proportionally with the magnitude of the conductor's motion (rotation in this scenario). At the same time, the reaction magnetic field caused by the eddy current itself is basically insignificant. However, with a continuous increase in rotational speed, this reaction magnetic field becomes more significant and interferes with the external magnetic field. Therefore, although the damping torque continues to increase, it does so at continually slower rate until the maximum damping torque is reached. In the second region, the interference between the external and reaction magnetic fields actually weakens the resultant flux density, hence the decreasing trend of the damping curve. The obtained torque-speed characteristics were found to agree well with the above description which can also be seen in an electromagnetic brake [14].

Considering that a decreasing trend in damping characteristic at high damper operating speeds is undesirable for suspension application, only the beginning region, from $0 \mathrm{rads}^{-1}$ to $100 \mathrm{rads}^{-1}$ in which the linear relationship was generally valid, was chosen for the determination of the rotational damping rate for the base design. Firstly, for eddy current damper involving ball-screw mechanism, first order (linear) curve-fitting of the said beginning region, considering interception at the point of origin to satisfy typical requirement of a damping curve, resulted in a rotational damping rate (gradient) of $0.0232 \mathrm{Nms}^{-r^{-1}}$ with a high $R^{2}$ value of 0.9851 (Figure 4(a)). Meanwhile, for the case with rack-andpinion mechanism, the same first order curve-fitting of the relevant beginning region gave a rotational damping rate of 0.0464
Nms.rad ${ }^{-1}$, with a similarly high $R^{2}$ value of 0.9735 which indicated an accurate capture of the damping curve (Figure 4(b)).

Due to the rotational-to-translational conversion mechanisms originated from the inerter, the rotational damping effect from the rotary eddy current damper model can be converted to the translational or linear damping as seen in a vehicle suspension, with the linear damping rate abiding the following direct relations respectively for ball-screw mechanism and rack-and-pinion mechanism adopted in this study:

$c_{\text {translational }}=(2 \pi / p)^{2} c_{\text {rotational }}$

$c_{\text {translational }}=\left(2 / d_{p}\right)^{2} c_{\text {rotational }}$

in which $c_{\text {rotational }}$ and $c_{\text {translational }}$ are the rotational damping rate and the corresponding translational or linear damping rate. Using the predetermined values of pitch and pinion diameter during the fulfillment of inertance, conversion back to the translational motion resulted in a linear damping rate of $228975 \mathrm{Nsm}^{-1}$ for eddy current damper involving ball-screw mechanism, and a notably different rate of $464 \mathrm{Nsm}^{-1}$ for the case with rack-and-pinion mechanism. For the purpose of an initial comparison, the suspension damping of the passenger vehicle used in this study, according to the specific vehicle parameter value from the quarter vehicle model, has a rate of $c=1500 \mathrm{Nsm}^{-1}$. Similarly, a past study on semi-active suspension damper which tested the same passenger vehicle adopted $900 \mathrm{Nsm}^{-1}$ and $1500 \mathrm{Nsm}^{-1}$ as the discrete switchable damping rates for the semi-active damper [19]. To add to these, the linear eddy current damper feasibility study [10] mentioned earlier also stated that the required mean damping rate in the range from $1500 \mathrm{Nsm}^{-1}$ to $2000 \mathrm{Nsm}^{-1}$ is acceptable for a passive suspension damper of a midsize vehicle. Judging from the above, the linear damping rate generated from the ball-screwbased eddy current damper seemed to be unsuitably high for the application in passenger vehicle suspension, while the damping rate generated from the eddy current damper with rack-and-pinion mechanism seemed to be applicable based on the similar order of magnitude to typical suspension damping requirement. However, these can only be confirmed by considering as well the results of parametric variations. The obtained damping characteristics due to parametric variations are shown collectively as Figure 5 (involving ball-screw mechanism) and Figure 6 (involving rack-andpinion mechanism), while the corresponding rotational and linear damping rates for cases with both mechanisms are summarized in Table 3.

Basically, the capability of these electromagnetic-related parametric variations to produce different damping rates lies in the fact that these parameters have notable effects on the magnetic flux density which, in turn, has an effect on eddy current induction. As a general observation, parametric variations which intensified the external magnetic field notably, such as a greater number of magnet segments, an increase in magnet height and a decrease in air gap, caused a greater achievable damping rate. In addition to this, some other specific observations are also worth discussing here. First of all, it was noted that the case with complete magnetic coverage actually gave negligible rotational damping instead of improving the situation. That was because the applied magnetic field in the case of a complete coverage was symmetrical about the axis of conductor's rotation, and this prevented the flow of eddy current. Conversely, eddy current will be generated if there is asymmetry in the magnetic field about the rotational axis [20]. Secondly, it was observed that the magnet configuration with opposite poles facing each other (mostly axially-oriented magnetic field) generated significant eddy current damping relative to the much reduced damping from the alternative configuration with like poles facing each other (mostly radially-oriented magnetic field). This highlighted the importance of having suitable magnetic field orientation, in addition to the requirement of being perpendicular to the conductor's motion, to maximize the generated opposing damping torque in a rotary eddy current damper. Finally, it was noted that both arc and circular magnet shapes produced 
approximately the same achievable damping, with the former slightly superior to the latter. Although this observation is also dependent on the dimensional side of things, in general the slightly greater damping due to the former shape was because a greater portion of the magnetic flux was applied near the outer edge of the flywheel conductor, hence the greater opposing torque. This is relevant specifically to a rotary eddy current damper as opposed to a linear counterpart.

Lastly, in terms of quantitative evaluation, for the rotary eddy current damper model considering ball-screw mechanism, it was found that the range of achievable linear damping rates, from 4935 $\mathrm{Nsm}^{-1}$ to as much as $826086 \mathrm{Nsm}^{-1}$, was not applicable to passenger vehicle suspension. However, the unsuitability was actually due to the ball-screw rotational-to-translational conversion rather than the eddy current damping itself. As a typical ball-screw's pitch is in the range of millimeters $(0.002 \mathrm{~m}$ in this case $)$, the conversion ratio as in (8) is usually inappropriately large. Therefore, even though the generated rotary eddy current damping seemed reasonable, the converted linear damping effect was inappropriately enlarged such that it became not applicable to passenger vehicle suspension. Apart from this, the typical ball-screw mechanism's inappropriate magnitude of conversion ratio also resulted in a very narrow linear damping operating speed range from $0 \mathrm{~ms}^{-1}$ to 0.1 $\mathrm{ms}^{-1}\left( \pm 0.1 \mathrm{~ms}^{-1}\right.$ for both jounce and rebound directions), as translated from the working rotational speed range of $0 \mathrm{rads}^{-1}$ to 100 $\operatorname{rads}^{-1}$. This narrow linear operating speed range was also found to be unfavorable for vehicle suspension application, considering that a suspension damper in a passenger vehicle can possibly reach $\pm 1 \mathrm{~ms}^{-1}$ during actual operation.

Conversely, the outlook for rotary eddy current damper considering rack-and-pinion mechanism was much brighter. The same parameter variations gave a range of achievable linear damping rates from negligible value up to $1535 \mathrm{Nsm}^{-1}$, which effectively resulted in good applicability in passenger vehicle suspension. It is worth noting here that the generated rotational eddy current damping rates were actually quite similar to those obtained from the cases with ball-screw mechanism; however, the notably different outlook was mainly down to the use of rack-and-pinion rotationalto-translational mechanism. With the pinion diameter typically in the range of centimeters $(0.020 \mathrm{~m}$ in this case), the resulted conversion ratio was much smaller, and the converted linear damping rates had the same order of magnitude as common suspension damping rates of passenger vehicles. Additionally, based on the same working rotational speed range, the translated linear damping operating speed range was determined to be from $0 \mathrm{~ms}^{-1}$ to 1 $\mathrm{ms}^{-1}$ ( $\pm 1 \mathrm{~ms}^{-1}$ for both jounce and rebound directions), which nicely matched the possible suspension damper speed during actual operation as suggested above.

Table 3: Summary of the Achievable Damping Rates for the Rotary Eddy Current Damper Model

\begin{tabular}{|c|c|c|c|c|}
\hline $\begin{array}{l}\text { Parameter Varia- } \\
\text { tion }\end{array}$ & $\begin{array}{l}\text { (Ball-screw) Rotational } \\
\text { Damping Rate, } c_{\text {rotational }} \\
\left(\mathrm{Nms}^{2} \mathrm{dad}^{-1}\right)\end{array}$ & $\begin{array}{l}\text { (Ball-screw) Linear Damp- } \\
\text { ing Rate, } c_{\text {translational }}\left(\mathrm{Nsm}^{-1}\right)\end{array}$ & $\begin{array}{c}\text { (Rack-and-pinion) Rotational } \\
\text { Damping Rate, } c_{\text {rotational }} \\
\left(\text { Nms.rad }^{-1}\right) \\
\end{array}$ & $\begin{array}{l}\text { (Rack-and-pinion) Linear } \\
\text { Damping Rate, } c_{\text {translational }} \\
\left(\mathrm{Nsm}^{-1}\right)\end{array}$ \\
\hline \multicolumn{5}{|l|}{ Air gap } \\
\hline $2 \mathrm{~mm}$ & 0.0232 & 228975 & 0.0464 & 464 \\
\hline $4 \mathrm{~mm}$ & 0.0152 & 150018 & 0.0343 & 343 \\
\hline $6 \mathrm{~mm}$ & 0.0101 & 99683 & 0.0208 & 208 \\
\hline $8 \mathrm{~mm}$ & 0.0085 & 83892 & 0.0148 & 148 \\
\hline $10 \mathrm{~mm}$ & 0.0051 & 50335 & 0.0117 & 117 \\
\hline \multicolumn{5}{|l|}{$\begin{array}{l}\text { Magnet configura- } \\
\text { tion }\end{array}$} \\
\hline Opposite poles & 0.0232 & 228975 & 0.0464 & 464 \\
\hline Like poles & 0.0008 & 7896 & 0.0013 & 13 \\
\hline Single pole & 0.0077 & 75996 & 0.0114 & 114 \\
\hline \multicolumn{5}{|l|}{$\begin{array}{l}\text { Coverage of mag- } \\
\text { net segment }\end{array}$} \\
\hline Partial & 0.0232 & 228975 & 0.0464 & 464 \\
\hline Complete & 0.0005 & 4935 & 0.0009 & 9 \\
\hline \multicolumn{5}{|l|}{ Magnet material } \\
\hline $\begin{array}{l}\text { Neodymium-based } \\
(\mathrm{NdFeB})\end{array}$ & 0.0232 & 228975 & 0.0464 & 464 \\
\hline $\begin{array}{l}\text { Samarium-Cobalt } \\
(\mathrm{SmCo})\end{array}$ & 0.0188 & 185549 & 0.0377 & 377 \\
\hline \multicolumn{5}{|l|}{ Height of magnet } \\
\hline $5 \mathrm{~mm}$ & 0.0102 & 100670 & 0.0217 & 217 \\
\hline $10 \mathrm{~mm}$ & 0.0232 & 228975 & 0.0464 & 464 \\
\hline $15 \mathrm{~mm}$ & 0.0319 & 314840 & 0.0747 & 747 \\
\hline $20 \mathrm{~mm}$ & 0.0408 & 402680 & 0.0845 & 845 \\
\hline \multicolumn{5}{|l|}{$\begin{array}{l}\text { Number of magnet } \\
\text { segment }\end{array}$} \\
\hline 1 segment & 0.0232 & 228975 & 0.0464 & 464 \\
\hline 2 segments & 0.0499 & 492493 & 0.0890 & 890 \\
\hline 4 segments & 0.0837 & 826086 & 0.1535 & 1535 \\
\hline \multicolumn{5}{|l|}{ Magnet shape } \\
\hline Arc-segment & 0.0232 & 228975 & 0.0464 & 464 \\
\hline Circular & 0.0222 & 219105 & 0.0408 & 408 \\
\hline
\end{tabular}




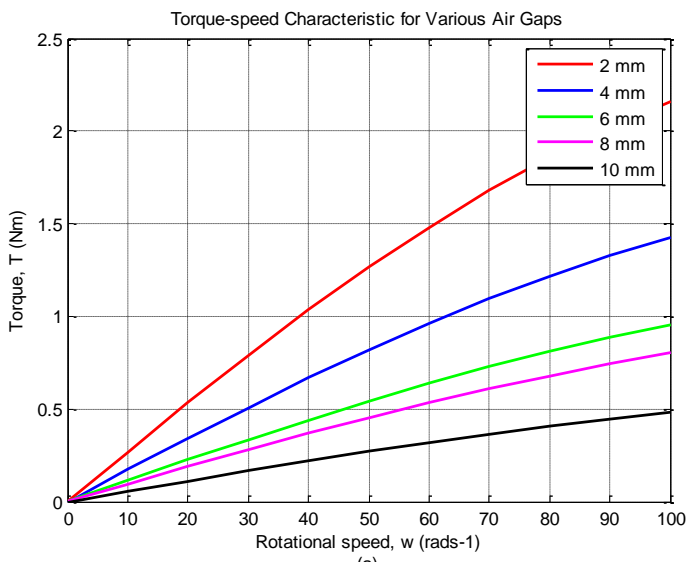

(a)

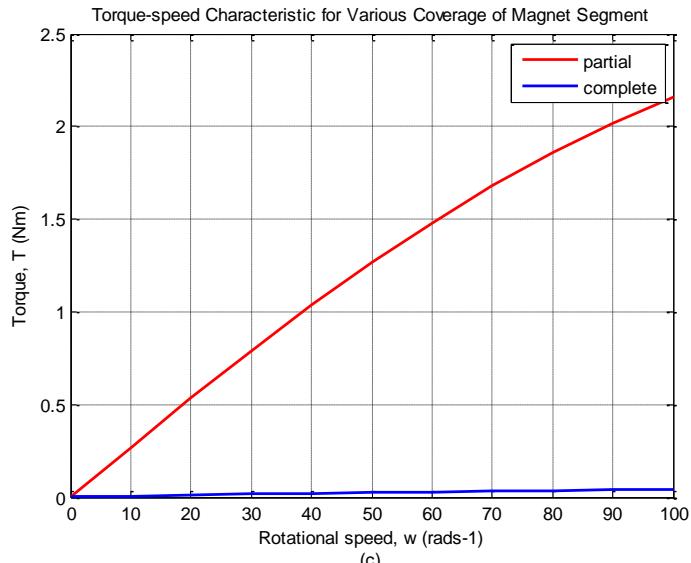

(c)

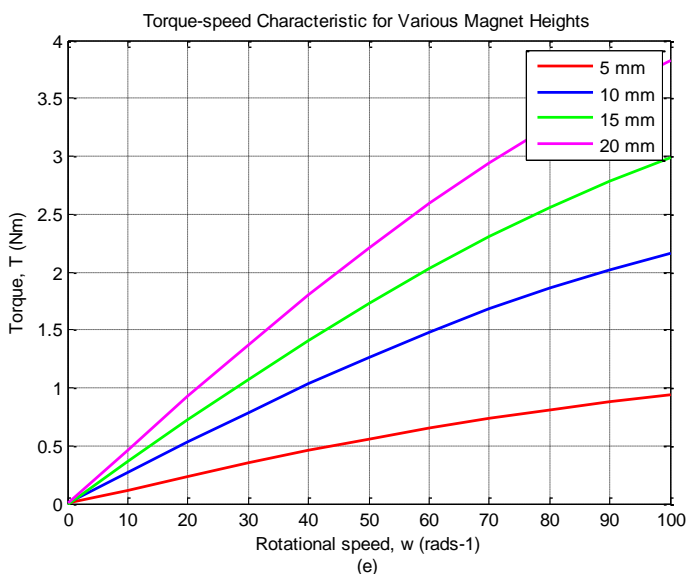

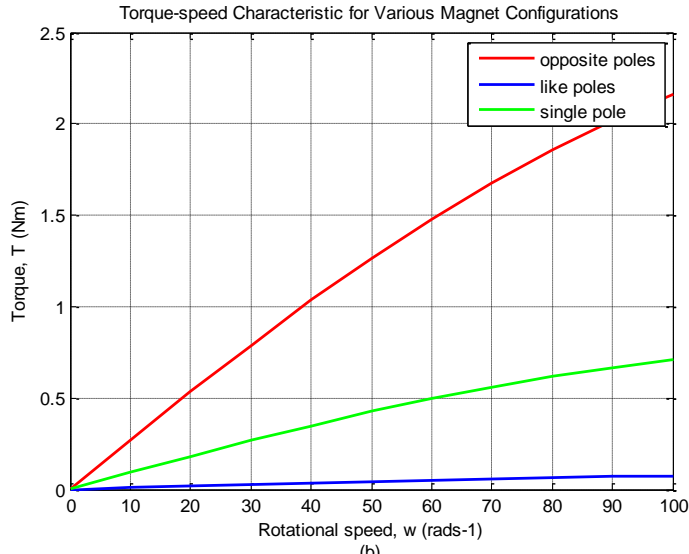
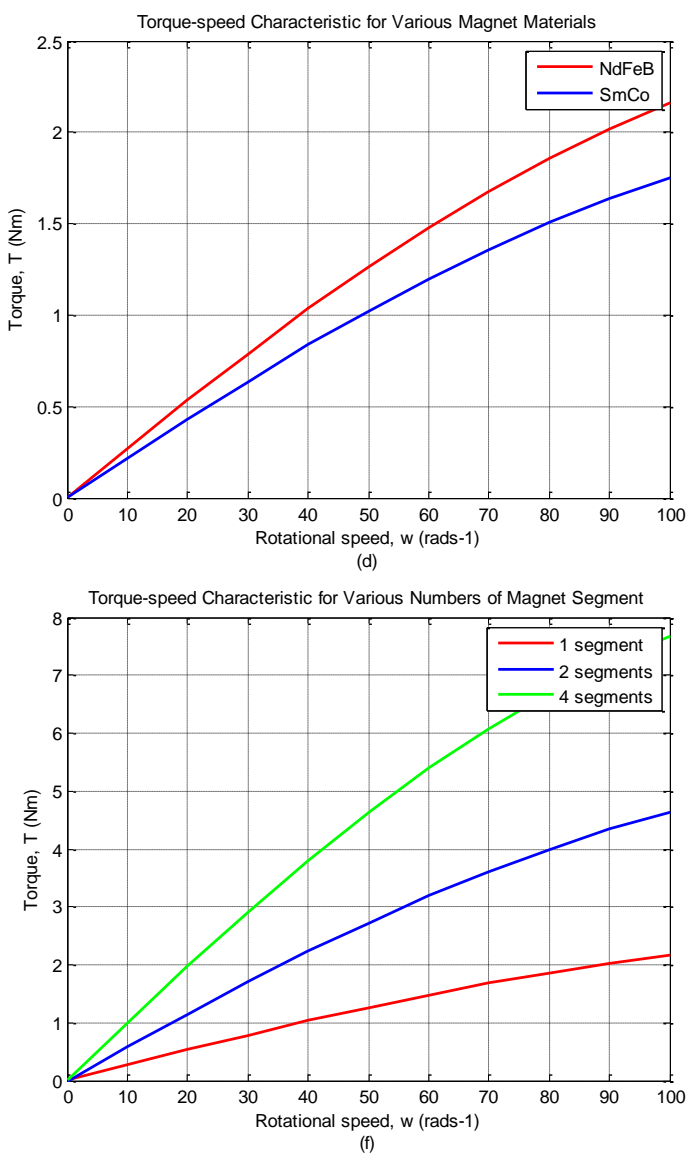

(f)

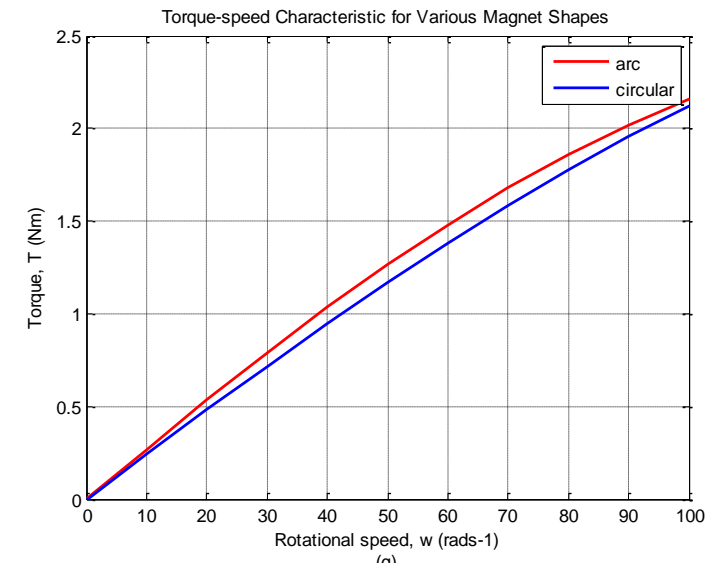

(g)

Fig. 5: Torque-speed characteristics for rotary eddy current damper involving ball-screw mechanism, due to variations in (a) air gap, (b) magnet configuration, (c) coverage of magnet, (d) magnet material, (e) height of magnet, (f) number of magnet segments, and (g) magnet shape 

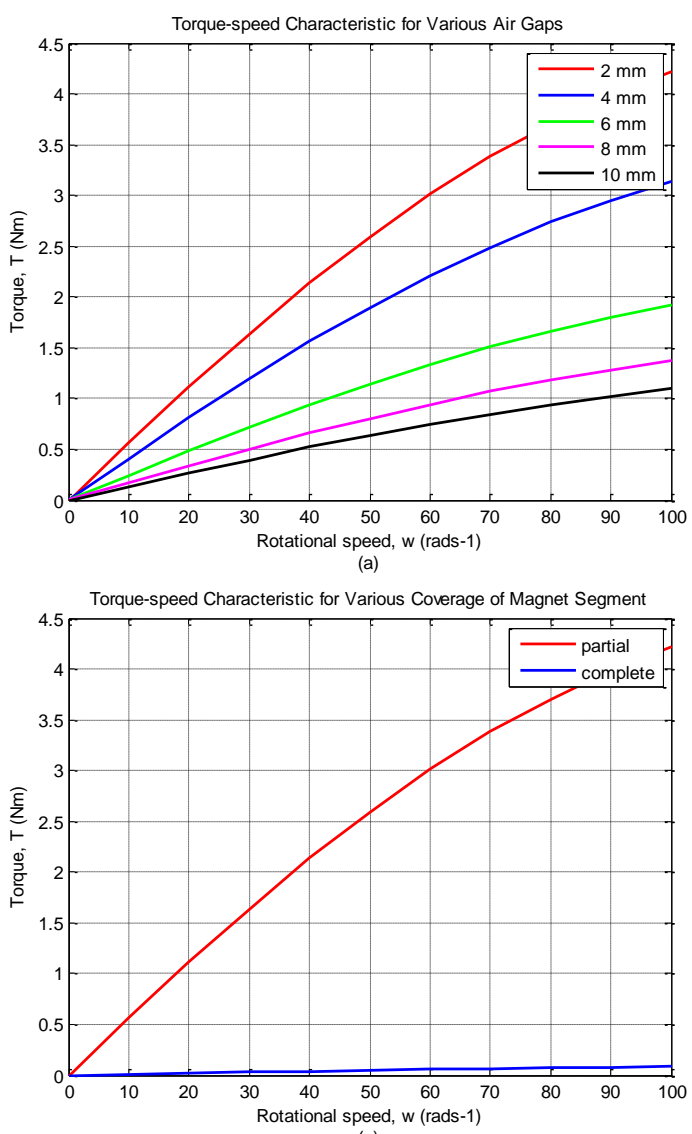

(c)

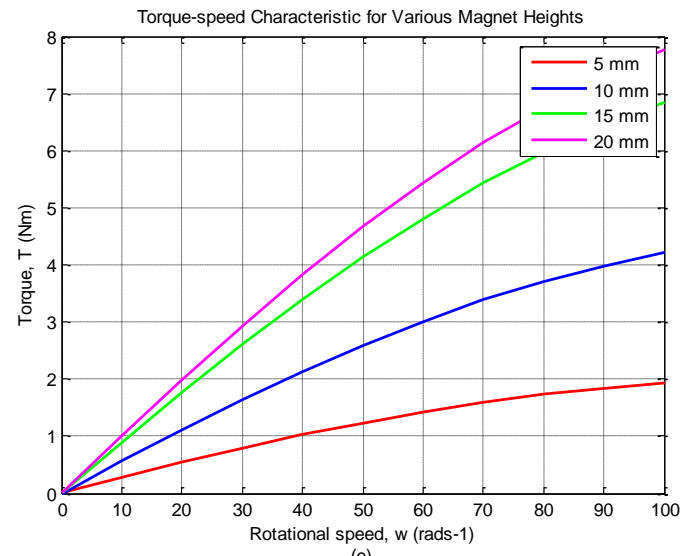

(e)
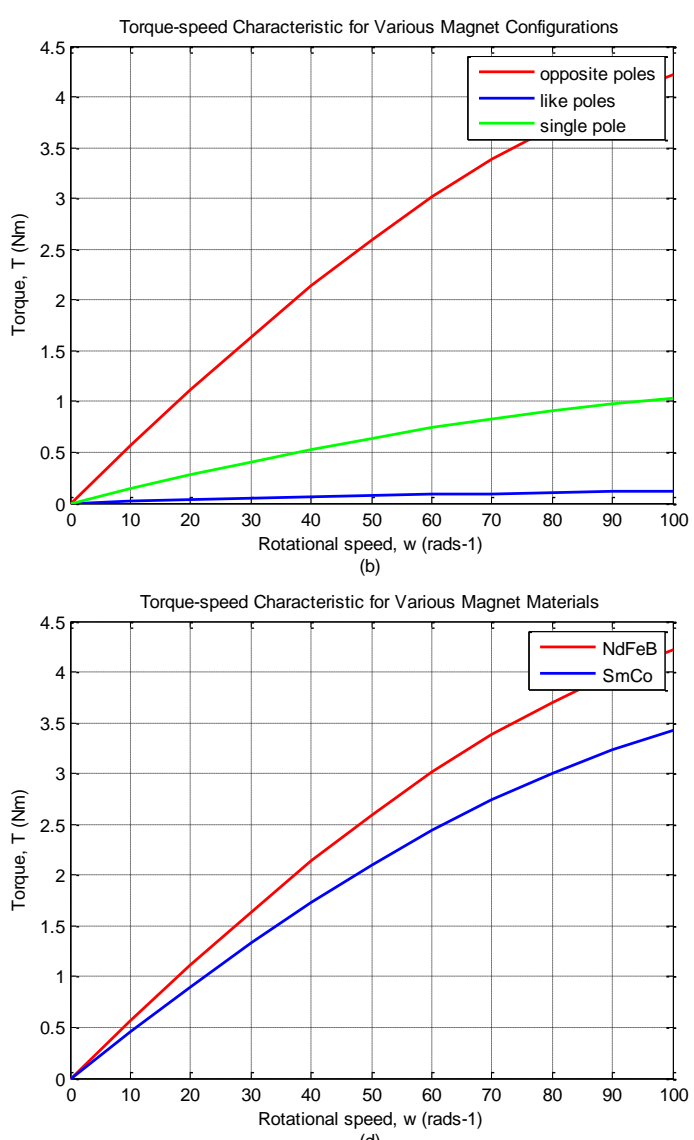
(d)

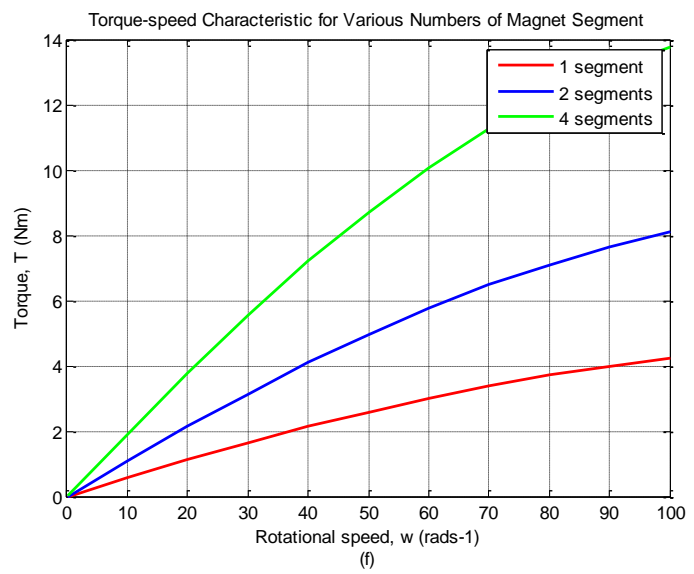

(f)

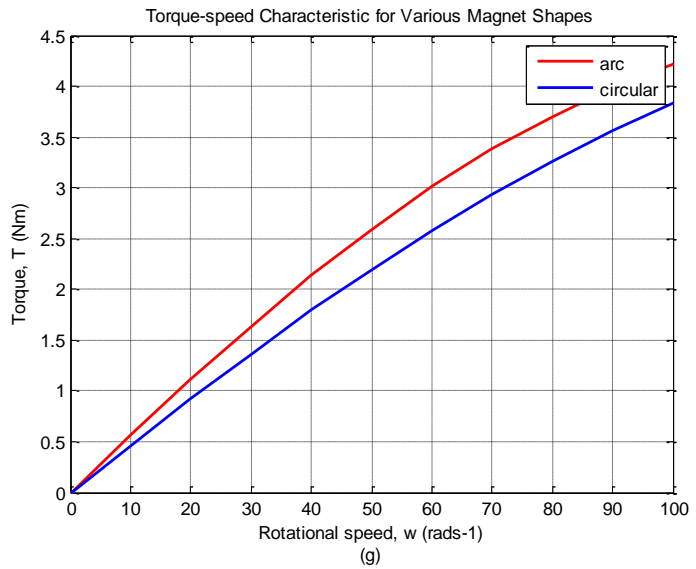

Fig. 6: Torque-speed characteristics for rotary eddy current damper involving rack-and-pinion mechanism, due to variations in (a) air gap, (b) magnet configuration, (c) coverage of magnet, (d) magnet material, (e) height of magnet, (f) number of magnet segments, and (g) magnet shape 


\section{Conclusion}

Summing from the above, it can be concluded that the applicability of rotary eddy current damper exists in passenger vehicle suspension that considers the inerter. The parametric analysis has shown that the generated damping effect due to eddy current induction can be intensified by variations of several electromagnetic-related parameters that basically intensify the magnetic flux density acting at the flywheel conductor, the most prominent ones being an increase in magnet segments, increase in magnet height, and reduction of air gap between magnet and conductor. More importantly, the parametric analysis has also confirmed that, together with suitable rotational-to-translational conversion mechanism of an inerter, a linear suspension damping rate of up to approximately $1500 \mathrm{Nsm}^{-1}$, which is suitable for passenger vehicle suspension application, can be achieved from certain individual parametric variation from the base design. Moreover, greater damping rates can expectedly be obtained by multiple parametric variations simultaneously, due to these parameters being independent to one another in general. With the achievable damping rates falling within those commonly used in passenger vehicle suspension, the applicability of rotary eddy current damper is therefore confirmed. Seeing the successful incorporation of passive eddy current damping in an inerter to have a practical parallel inerter suspension layout, it is hoped that this study will inspire further implementations of this suspension layout, such as a semiactive suspension employing parallel inerter, with variable eddy current damping through variable instead of constant magnetic field.

\section{Acknowledgement}

This work was supported by PPP Research Grant, (PV051-2011A) and Equitable Society Research Cluster (ESRC), University of Malaya (RP006C-13 SBS). The authors thank University of Malaya for the facilities and supports that has led to the completion of this research.

\section{References}

[1] Soong MF, Ramli R \& Mahadi WNL, "Using gear mechanism in vehicle suspension as a method of altering suspension characteristic", Journal of Vibration and Control, Vol.21, No.11, (2015), pp.2187-2199.

[2] Li C, Liang M, Wang Y \& Dong Y, "Vibration suppression using two-terminal flywheel. Part II: Application to vehicle passive suspension", Journal of Vibration and Control, Vol.18, No.9, (2012), pp.1353-1365.

[3] Scheibe F \& Smith MC, "Analytical solutions for optimal ride comfort and tyre grip for passive vehicle suspensions", Vehicle System Dynamics, Vol.47, No.10, (2009), pp.1229-1252.

[4] Smith MC \& Wang FC, "Performance benefits in passive vehicle suspensions employing inerters", Vehicle System Dynamics, Vol.42, No.4, (2004), pp.235-257.

[5] Soong MF, Ramli R \& Mahadi WNL, "Vehicle suspensions with parallel inerter: Effectiveness in improving vibration isolation", Journal of Vibroengineering, Vol.16, No.1, (2014), pp.256-265.

[6] Soong MF, Ramli R, Mahadi WNL \& Saifizul A, "Ride improvement of vehicle suspensions with switchable inerter based on force cancellation strategy", Journal of Vibroengineering, Vol.19, No.2, (2017), pp.1260-1272.

[7] Zhang X, Ahmadian M \& Guo K, "On the benefits of semi-active suspensions with inerters", Shock and Vibration, Vol.19, No.3, (2012), pp.257-272.

[8] Smith MC, "Synthesis of mechanical networks: The inerter", IEEE Transactions on Automatic Control, Vol.47, No.10, (2002), pp.1648-1662.

[9] Papageorgiou C, Houghton NE \& Smith MC, "Experimental testing and analysis of inerter devices", Journal of Dynamic Systems, Measurement, and Control - Transactions of the ASME, Vol.131, No.1, 011001, (2009), pp.1-11.
[10] Ebrahimi B, Khamesee MB \& Golnaraghi F, "Eddy current damper feasibility in automobile suspension: Modeling, simulation and testing”, Smart Materials and Structures, Vol.18, No.1, 015017, (2009), pp.1-12.

[11] Li P \& Zuo L, "Influences of the electromagnetic regenerative dampers on the vehicle suspension performance", Proceedings of the Institution of Mechanical Engineers Part D - Journal of Automobile Engineering, Vol.231, No.3, (2017), pp.383-394.

[12] Maddah AA, Hojjat Y, Karafi MR \& Ashory MR, "Reduction of magneto rheological dampers stiffness by incorporating of an eddy current damper", Journal of Sound and Vibration, Vol.396, (2017), pp.51-68.

[13] Sodano HA, Bae JS, Inman DJ \& Belvin WK, "Concept and model of eddy current damper for vibration suppression of a beam", Journal of Sound and Vibration, Vol.288, No.4-5, (2005), pp.1177-1196.

[14] Gay SE, Contactless magnetic brake for automotive applications, Doctoral Dissertation, (2005). http://repository.tamu.edu/bitstream/handle/1969.1/ETD-TAMU1005/GAY-DISSERTATION.pdf

[15] Sodano HA, Bae JS, Inman DJ \& Belvin WK, "Improved concept and model of eddy current damper", Journal of Vibration and Acoustics - Transactions of the ASME, Vol.128, No.3, (2006), pp.294-302.

[16] Sodano HA, Inman DJ \& Belvin WK, "New semi-active damping concept using eddy currents", Proceedings of the SPIE, Vol.5760, (2005), pp. 293-304

[17] Sodano HA \& Inman DJ, "Non-contact vibration control system employing an active eddy current damper", Journal of Sound and Vibration, Vol.305, No.4-5, (2007), pp.596-613.

[18] Ebrahimi B, Bolandhemmat H, Khamesee MB \& Golnaraghi F, “A hybrid electromagnetic shock absorber for active vehicle suspension systems", Vehicle System Dynamics, Vol.49, No.1-2, (2011), pp.311-332.

[19] Sharma K, Crolla DA \& Wilson DA, "Derivation of a control law for a 3 state switchable damper suspension system for improving road vehicle ride characteristics", International Symposium on Theory of Machines and Mechanisms, (1992).

[20] Montgomery H, "Current flow patterns in a faraday disc", European Journal of Physics, Vol.25, No.2, (2004), pp.171-183. 